\title{
Solutions of Fractional Konopelchenko-Dubrovsky and Nizhnik-Novikov-Veselov Equations Using a Generalized Fractional Subequation Method
}

\author{
Yanqin Liu ${ }^{1,2}$ and Limei Yan ${ }^{1}$ \\ ${ }^{1}$ School of Mathematical Sciences, Dezhou University, Dezhou 253023, China \\ ${ }^{2}$ The Center of Data Processing and Analyzing, Dezhou University, Dezhou 253023, China \\ Correspondence should be addressed to Yanqin Liu; yanqinliu@dzu.edu.cn
}

Received 13 July 2013; Accepted 8 August 2013

Academic Editor: Juan J. Trujillo

Copyright (C) 2013 Y. Liu and L. Yan. This is an open access article distributed under the Creative Commons Attribution License, which permits unrestricted use, distribution, and reproduction in any medium, provided the original work is properly cited.

A new generalized fractional subequation method based on the relationship of fractional coupled equations is proposed. This method is applied to the space-time fractional coupled Konopelchenko-Dubrovsky equations and Nizhnik-Novikov-Veselov equations. As a result, many exact solutions are obtained including hyperbolic function solutions, trigonometric function solutions, and rational solutions. It is observed that the proposed approach provides a simple and reliable tool for solving many other fractional coupled differential equations.

\section{Introduction}

Fractional calculus is one of the generalizations of ordinary calculus. Generally speaking, there are two kinds of fractional derivatives. One is nonlocal fractional derivative $[1,2]$, that is, Caputo derivative and Riemann-Liouville derivative which have been used successfully in various fields of science and engineering. The other one is the local fractional derivative, that is, Kolwankar-Gangal (K-G) derivative $[3,4]$, Chen's fractal derivative [5, 6], Cresson's derivative [7], and Jumarie's modified Riemann-Liouville derivative [8]. At the same time, fractional differential equations have attracted much attention in a variety of applied sciences. However, we have difficulty in finding exact analytical solutions [9-12] of fractional differential equations that appear more and more frequently in different research areas and engineering applications. So, numerical methods have been used to handle these equations, and some semianalytical techniques [13-16] have also largely been used to solve these equations.

Based on homogeneous balance principle [17], Jumarie's modified Riemann-Liouville derivative [8], and symbolic computation, S. Zhang and H.-Q. Zhang proposed a fractional subequation method to search for explicit solutions of FDEs. By using this method, S. Zhang and H.-Q. Zhang successfully obtained some exact solutions of space-time fractional biological population model and fractional Fokas equation [18]. Jafari et al. have given some solutions of the fractional Cahn-Hilliard and Klein-Gordon equations [19]. Tang et al. [20] proposed a generalized fractional subequation method for fractional differential equations with variable coefficients. Guo et al. [21] and Zhao et al. [22] both improved the fractional subequation and applied to space-time fractional coupled differential equations; in their paper, they choose two or three appropriate ansätz. However, for some coupled equations $[23,24]$, even some fractional coupled equations, we can get the relationship of the functions. So, we propose a new generalized fractional subequation which chooses only one appropriate ansätz and use this method to solve the following two NFDEs.

(1) The space-time fractional coupled KonopelchenkoDubrovsky (KD) equations in the form

$$
\begin{gathered}
D_{t}^{\alpha} u-D_{x}^{3 \alpha} u-6 b u D_{x}^{\alpha} u+\frac{3}{2} a^{2} u^{2} D_{x}^{\alpha} u-3 D_{y}^{\alpha} v+3 a D_{x}^{\alpha} u v=0, \\
D_{y}^{\alpha} u=D_{x}^{\alpha} v,
\end{gathered}
$$


which is a transformed generalization of the $\mathrm{KD}$ equations [25], where $a$ and $b$ are real constants. Equation (1) is a fractional evolution equation on two spatial dimensions and one temporal, where $x$ and $y$ are the running coordinates, $t$ is the time, and $u=u(x, y, t)$ and $v=v(x, y, t)$ are the amplitudes of the relevant waves. $D_{t}^{\alpha}(\cdot)$ and $D_{x}^{\alpha}(\cdot)$ are Jumarie's modified Riemann-Liouville derivative of order $\alpha$ defined in Section 2, $0<\alpha \leq 1$. The Jumarie's modified Riemann-Liouville derivative has many interesting properties. The KD equations can be used to describe the ocean dynamics, fluid mechanics, and plasma physics, and the Gardner, KP, modified KP, and $\mathrm{KD}$ equations are all the special cases of (1). When $\alpha=1$, $u_{y}=0$, (1) is the Gardner equation (combined $\mathrm{KdV}$ and modified equation). When $\alpha=1, a=0$, (1) is the wellknown Kadomtsev-Petviashvili (KP) equation, and modified KP equation reads from (1) for $\alpha=1, b=0$.

(2) The space-time fractional coupled Nizhnik-NovikovVeselov (NNV) equation in the form

$$
\begin{gathered}
D_{t}^{\alpha} u=A D_{x}^{3 \alpha} u+B D_{y}^{3 \alpha} u-3 A u D_{x}^{\alpha} v-3 A v D_{x}^{\alpha} u \\
-3 B u D_{y}^{\alpha} w-3 B w D_{y}^{\alpha} u, \\
D_{x}^{\alpha} u=D_{y}^{\alpha} v, \\
D_{y}^{\alpha} u=D_{x}^{\alpha} w,
\end{gathered}
$$

where $0<\alpha \leq 1, A$ and $B$ are given constants satisfying $A+B \neq 0$, and $u, v$, and $w$ are the functions of $(x, y, t)$, the case when $\alpha \rightarrow 1$ was studied in [26]. NNV equations have been studied over several areas of physics including condense matter physics, fluid mechanics, plasma physics, and optics.

The rest of this paper is organized as follows. In Section 2, some basic definitions of Jumarie's modified RiemannLiouville derivative and the main steps of the generalized fractional subequation method are given. In Section 3, we construct the exact solutions of above space-time fractional coupled equations via this new generalized method. Some conclusions and discussions are shown in Section 4.

\section{Jumarie's Modified Riemann-Liouville Derivative and Generalized Fractional Subequation Method}

The Jumarie's modified Riemann-Liouville derivative [8] of order $\alpha$ time-fractional derivative operator of order $\alpha>0$ is defined as

$$
D_{t}^{\alpha} f=\left\{\begin{array}{r}
\frac{1}{\Gamma(1-\alpha)} \int_{0}^{t}(t-\xi)^{-\alpha-1}(f(\xi)-f(0)) d \xi \\
\alpha<0, \\
\frac{1}{\Gamma(1-\alpha)} \frac{d}{d t} \int_{0}^{t}(t-\xi)^{-\alpha}(f(\xi)-f(0)) d \xi \\
\left(f^{(n)}(t)\right)^{\alpha-n}, \quad n \leq \alpha<n+1, n \geq 1 .
\end{array}\right.
$$

Some properties for the proposed modified RiemannLiouville derivative are listed in [8] as follows:

$$
\begin{gathered}
D_{t}^{\alpha} t^{\delta}=\frac{\Gamma(1+\delta)}{\Gamma(1+\delta-\alpha)} t^{\delta-\alpha}, \quad \delta>0, \\
D_{t}^{\alpha}(f(t) g(t))=g(t) D_{t}^{\alpha} f(t)+f(t) D_{t}^{\alpha} g(t), \\
D_{t}^{\alpha} f[g(t)]=f_{g}^{\prime}[g(t)] D_{t}^{\alpha} g(t), \\
D_{t}^{\alpha} f[g(t)]=D_{g}^{\alpha} f[g(t)]\left(g^{\prime}(t)\right)^{\alpha} .
\end{gathered}
$$

The above equations play an important role in fractional calculus in the following sections.

we propose a generalized fractional subequation method; the essential steps of this method are described as follows.

Step 1. Suppose that NFDEs with independent variables $X=$ $\left(x_{1}, x_{2}, x_{3}, \ldots, x_{m}, t\right)$ are given by

$$
\begin{gathered}
P\left(u, v, u_{t}, u_{x_{1}}, \ldots, v_{t}, v_{x_{1}}, \ldots, D_{t}^{\alpha} u, D_{x_{1}}^{\alpha} u, \ldots,\right. \\
\left.D_{t}^{\alpha} v, D_{x_{1}}^{\alpha} v, \ldots\right)=0, \quad 0<\alpha \leq 1, \\
Q\left(u, v, u_{t}, u_{x_{1}}, \ldots, v_{t}, v_{x_{1}}, \ldots, D_{t}^{\alpha} u, D_{x_{1}}^{\alpha} u, \ldots,\right. \\
\left.D_{t}^{\alpha} v, D_{x_{1}}^{\alpha} v, \ldots\right)=0, \quad 0<\alpha \leq 1,
\end{gathered}
$$

where $D_{t}^{\alpha}(\cdot)$ and $D_{x_{1}}^{\alpha}(\cdot)$ are Jumarie's modified RiemannLiouville derivative with respect to $t$ and $x_{1}, u=u(x, t)$, $v=v(x, t)$ are unknown functions, $P$ is a polynomial in $u, v$, and their various partial derivatives, $Q$ is a polynomial in $u, v$, and their various partial derivatives, and the highest order derivatives and nonlinear terms are involved.

Step 2. By using the traveling wave transformations

$$
\begin{gathered}
u\left(x_{1}, \ldots, x_{m}, t\right)=u(\xi), \quad v\left(x_{1}, \ldots, x_{m}, t\right)=v(\xi) \\
\xi=k_{1} x_{1}+\cdots+k_{m} x_{m}+c t
\end{gathered}
$$

where $c$ is a constant to be determined later, the NFDE (7) is reduced to the following nonlinear fractional ordinary differential equation (ODE) for $u(\xi)$ and $v(\xi)$ :

$$
\begin{aligned}
& P\left(u, v, c u^{\prime}, k_{1} u^{\prime}, \ldots, c v^{\prime}, k_{1} v^{\prime}, \ldots, c^{\alpha} D_{\xi}^{\alpha} u, k_{1}^{\alpha} D_{\xi}^{\alpha} u, \ldots,\right. \\
& \left.c^{\alpha} D_{\xi}^{\alpha} v, k_{1}^{\alpha} D_{\xi}^{\alpha} v, \ldots\right)=0, \\
& Q\left(u, v, c u^{\prime}, k_{1} u^{\prime}, \ldots, c v^{\prime}, k_{1} v^{\prime}, \ldots, c^{\alpha} D_{\xi}^{\alpha} u, k_{1}^{\alpha} D_{\xi}^{\alpha} u, \ldots,\right. \\
& \left.c^{\alpha} D_{\xi}^{\alpha} v, k_{1}^{\alpha} D_{\xi}^{\alpha} v, \ldots\right)=0,
\end{aligned}
$$

Step 3. For some coupled equations, we get the relationship

$$
v=f(u),
$$

and substituting into (8), one has

$$
\begin{gathered}
Q\left(u, v, c u^{\prime}, k_{1} u^{\prime}, \ldots, c v^{\prime}, k_{1} v^{\prime}, \ldots, c^{\alpha} D_{\xi}^{\alpha} u, k_{1}^{\alpha} D_{\xi}^{\alpha} u, \ldots,\right. \\
\left.c^{\alpha} D_{\xi}^{\alpha} v, k_{1}^{\alpha} D_{\xi}^{\alpha} v, \ldots\right)=0 .
\end{gathered}
$$


Step 4. We suppose that (12) has the following solution:

$$
u(\xi)=\sum_{i=-n}^{i=n} a_{i} \varphi^{i}
$$

where $a_{i}(i=-n,-n+1, \ldots, n-1, n)$ are constants to be determined later, $n$ is a positive integer determined by balancing the highest order derivatives and nonlinear terms in (12) (see [17] for details), and $\varphi=\varphi(\xi)$ satisfies the following fractional Riccati equation:

$$
D_{\xi}^{\alpha} \varphi(\xi)=\sigma+\varphi^{2}(\xi)
$$

By using the generalized Exp-function method via MittagLeffler functions, S. Zhang and H.-Q. Zhang first obtained generalized hyperbolic and trigonometric functions of fractional Riccati equation [18], and the obtained five solutions of (14) are

$$
\varphi(\xi)= \begin{cases}-\sqrt{-\sigma} \tanh _{\alpha}(\sqrt{-\sigma} \xi), & \sigma<0, \\ -\sqrt{-\sigma} \operatorname{coth}_{\alpha}(\sqrt{-\sigma} \xi), & \sigma<0, \\ \sqrt{\sigma} \tan _{\alpha}(\sqrt{\sigma} \xi), & \sigma>0, \\ -\sqrt{\sigma} \cot _{\alpha}(\sqrt{\sigma} \xi), & \sigma>0, \\ -\frac{\Gamma(1+\alpha)}{\xi^{\alpha}+\omega}, \omega=\text { const., } & \sigma=0,\end{cases}
$$

where $\tanh _{\alpha}, \operatorname{coth}_{\alpha}, \tan _{\alpha}$, and $\cot _{\alpha}$ are generalized hyperbolic and trigonometric functions defined in [18] as

$$
\begin{gathered}
\tanh _{\alpha}(x)=\frac{\sinh _{\alpha}(x)}{\cosh _{\alpha}(x)}, \quad \operatorname{coth}_{\alpha}(x)=\frac{\cosh _{\alpha}(x)}{\sinh _{\alpha}(x)}, \\
\sinh _{\alpha}(x)=\frac{E_{\alpha}\left(x^{\alpha}\right)-E_{\alpha}\left(-x^{\alpha}\right)}{2}, \\
\cosh _{\alpha}(x)=\frac{E_{\alpha}\left(x^{\alpha}\right)+E_{\alpha}\left(-x^{\alpha}\right)}{2}, \\
\tan _{\alpha}(x)=\frac{\sin _{\alpha}(x)}{\cos _{\alpha}(x)}, \quad \cot _{\alpha}(x)=\frac{\cos _{\alpha}(x)}{\sin _{\alpha}(x)} \\
\sin _{\alpha}(x)=\frac{E_{\alpha}\left(i x^{\alpha}\right)-E_{\alpha}\left(-i x^{\alpha}\right)}{2 i} \\
\cos _{\alpha}(x)=\frac{E_{\alpha}\left(i x^{\alpha}\right)+E_{\alpha}\left(-i x^{\alpha}\right)}{2}
\end{gathered}
$$

where $E_{\alpha}(z)=\sum_{k=0}^{\infty}\left(z^{k} / \Gamma(1+k \alpha)\right)$ is the Mittag-Leffler function.

Step 5. Substituting (13) into (12) along with (14) and using the properties of Jumarie's modified Riemann-Liouville derivative $(4)-(7)$, we can get a polynomial in $\varphi(\xi)$. Setting all the coefficients of $\varphi^{k}(k=0,1,2, \ldots,-1,-2, \ldots)$ to zero yields a set of overdetermined nonlinear algebraic equations for $c, k_{i}(i=1,2, \ldots, m), a_{j}(j=-n,-n+1, \ldots, n-1, n)$.

Step 6. Take advantage of the known solutions of (14) to get the solutions of the fractional coupled NPDEs in concern.

\section{Solutions of Fractional Coupled KD Equation and NNV Equations}

In this section, we apply the generalized fractional subequation method for solving the NPDEs (1) and (2).

Example 1. The space-time fractional $\mathrm{KD}$ equations. By considering the traveling wave transformations $u=u(\xi), v=$ $v(\xi)$, and $\xi=l x+m y+n t$, (1) can be reduced to the following nonlinear fractional ODEs:

$$
\begin{gathered}
n^{\alpha} D_{\xi}^{\alpha} u-l^{3 \alpha} D_{\xi}^{3 \alpha} u-6 b l^{\alpha} u D_{\xi}^{\alpha} u+\frac{3}{2} a^{2} l^{\alpha} u^{2} D_{\xi}^{\alpha} u \\
-3 m^{\alpha} D_{\xi}^{\alpha} v+3 a l^{\alpha} v D_{\xi}^{\alpha} u=0, \\
m^{\alpha} D_{\xi}^{\alpha} u=l^{\alpha} D_{\xi}^{\alpha} v .
\end{gathered}
$$

From (18) and using the definition of Jumarie's modified Riemann-Liouville derivative, one gets

$$
v=\frac{m^{\alpha}}{l^{\alpha}} u+c
$$

where $c$ is the arbitrary constant. Substituting (19) into (17), one obtains

$$
\begin{aligned}
& \left(n^{\alpha}-6 b l^{\alpha} u+\frac{3}{2} a^{2} l^{\alpha} u^{2}-3 \frac{m^{2 \alpha}}{l^{\alpha}}+3 a m^{\alpha} u+3 a l^{\alpha} c\right) \\
& \times D_{\xi}^{\alpha} u-l^{3 \alpha} D_{\xi}^{3 \alpha} u=0 .
\end{aligned}
$$

By balancing the highest order derivative terms and nonlinear terms in (20), we suppose that (20) has the following formal solution:

$$
u(\xi)=a_{0}+a_{1} \varphi(\xi)+\frac{a_{2}}{\varphi(\xi)}
$$

Substituting (21) into (20) along with (14) and collecting the coefficients of $\varphi^{i}$ and setting them to be zero, we can get a set of algebraic equation about $l, m, n, c, a_{0}, a_{1}$, and $a_{2}$. Solving the algebraic equations by Mathematica, we have the following.

Case 1. One has

$$
\begin{gathered}
a_{0}=\frac{2 b-a m^{\alpha} l^{-\alpha}}{a^{2}}, \quad a_{1}=0, \quad a_{2}=-\frac{2 l^{\alpha} \sigma}{\sqrt{a}}, \\
n=\left(\left(-12 a b m^{\alpha}+9 a^{2} m^{2 \alpha} l^{-\alpha}+12 b^{2} l 6 \alpha-6 b a^{3} c l^{\alpha}\right.\right. \\
\left.\left.+4 a^{3} l^{3 \alpha} \sigma\right) \times\left(2 a^{2}\right)^{-1}\right)^{1 / \alpha} .
\end{gathered}
$$

Case 2. One has

$$
\begin{gathered}
a_{0}=\frac{2 b-a m^{\alpha} l^{-\alpha}}{a^{2}}, \quad a_{1}=0, \quad a_{2}=\frac{2 l^{\alpha} \sigma}{\sqrt{a}}, \\
n=\left(\left(-12 a b m^{\alpha}+9 a^{2} m^{2 \alpha} l^{-\alpha}+12 b^{2} l 6 \alpha\right.\right. \\
\left.\left.\quad-6 b a^{3} c l^{\alpha}+4 a^{3} l^{3 \alpha} \sigma\right) \times\left(2 a^{2}\right)^{-1}\right)^{1 / \alpha} .
\end{gathered}
$$


Case 3. One has

$$
\begin{gathered}
a_{0}=\frac{2 b-a m^{\alpha} l^{-\alpha}}{a^{2}}, \quad a_{1}=\frac{2 l^{\alpha}}{\sqrt{a}}, \quad a_{2}=0, \\
n=\left(\left(-12 a b m^{\alpha}+9 a^{2} m^{2 \alpha} l^{-\alpha}+12 b^{2} l 6 \alpha\right.\right. \\
\left.\left.\quad-6 b a^{3} c l^{\alpha}+4 a^{3} l^{3 \alpha} \sigma\right) \times\left(2 a^{2}\right)^{-1}\right)^{1 / \alpha} .
\end{gathered}
$$

Case 4. One has

$$
\begin{gathered}
a_{0}=\frac{2 b-a m^{\alpha} l^{-\alpha}}{a^{2}}, \quad a_{1}=-\frac{2 l^{\alpha}}{\sqrt{a}}, \quad a_{2}=0, \\
n=\left(\left(-12 a b m^{\alpha}+9 a^{2} m^{2 \alpha} l^{-\alpha}+12 b^{2} l 6 \alpha-6 b a^{3} c l^{\alpha}\right.\right. \\
\left.\left.+4 a^{3} l^{3 \alpha} \sigma\right) \times\left(2 a^{2}\right)^{-1}\right)^{1 / \alpha} .
\end{gathered}
$$

Case 5. One has

$$
\begin{gathered}
a_{0}=\frac{2 b-a m^{\alpha} l^{-\alpha}}{a^{2}}, \quad a_{1}=-\frac{2 l^{\alpha}}{\sqrt{a}}, \quad a_{2}=-\frac{2 l^{\alpha} \sigma}{\sqrt{a}} \\
n=\left(\left(-12 a b m^{\alpha}+9 a^{2} m^{2 \alpha} l^{-\alpha}+12 b^{2} l 6 \alpha-6 b a^{3} c l^{\alpha}\right.\right. \\
\left.\left.\quad-8 a^{3} l^{3 \alpha} \sigma\right) \times\left(2 a^{2}\right)^{-1}\right)^{1 / \alpha} .
\end{gathered}
$$

Case 6. One has

$$
\begin{gathered}
a_{0}=\frac{2 b-a m^{\alpha} l^{-\alpha}}{a^{2}}, \quad a_{1}=\frac{2 l^{\alpha}}{\sqrt{a}}, \quad a_{2}=-\frac{2 l^{\alpha} \sigma}{\sqrt{a}} \\
n=\left(\left(-12 a b m^{\alpha}+9 a^{2} m^{2 \alpha} l^{-\alpha}+12 b^{2} l 6 \alpha-6 b a^{3} c l^{\alpha}\right.\right. \\
\left.\left.+16 a^{3} l^{3 \alpha} \sigma\right) \times\left(2 a^{2}\right)^{-1}\right)^{1 / \alpha} .
\end{gathered}
$$

Case 7. One has

$$
\begin{gathered}
a_{0}=\frac{2 b-a m^{\alpha} l^{-\alpha}}{a^{2}}, \quad a_{1}=-\frac{2 l^{\alpha}}{\sqrt{a}}, \quad a_{2}=\frac{2 l^{\alpha} \sigma}{\sqrt{a}} \\
n=\left(\left(-12 a b m^{\alpha}+9 a^{2} m^{2 \alpha} l^{-\alpha}+12 b^{2} l 6 \alpha-6 b a^{3} c l^{\alpha}\right.\right. \\
\left.\left.\quad+16 a^{3} l^{3 \alpha} \sigma\right) \times\left(2 a^{2}\right)^{-1}\right)^{1 / \alpha} .
\end{gathered}
$$

Case 8. One has

$$
\begin{gathered}
a_{0}=\frac{2 b-a m^{\alpha} l^{-\alpha}}{a^{2}}, \quad a_{1}=\frac{2 l^{\alpha}}{\sqrt{a}}, \quad a_{2}=\frac{2 l^{\alpha} \sigma}{\sqrt{a}}, \\
n=\left(\left(-12 a b m^{\alpha}+9 a^{2} m^{2 \alpha} l^{-\alpha}+12 b^{2} l 6 \alpha-6 b a^{3} c l^{\alpha}\right.\right. \\
\left.\left.\quad-9 a^{3} l^{3 \alpha} \sigma\right) \times\left(2 a^{2}\right)^{-1}\right)^{1 / \alpha} .
\end{gathered}
$$

Using Case 1, (21), and the solutions of (14), we can find the following exact solutions of NFDEs (1):

$$
\begin{gathered}
u_{1}=-\frac{2 \sqrt{-\sigma} l^{\alpha}}{\sqrt{a} \tanh _{\alpha}(-\sqrt{-\sigma} \xi)}+\frac{2 b l^{\alpha}-a m^{\alpha}}{a^{2} l^{\alpha}}, \\
v_{1}=-\frac{2 \sqrt{-\sigma} m^{\alpha}}{\sqrt{a} \tanh _{\alpha}(-\sqrt{-\sigma} \xi)}+\frac{2 b m^{\alpha} l^{\alpha}-a m^{2 \alpha}}{a^{2} l^{2 \alpha}}+c,
\end{gathered}
$$

where $\sigma<0, \xi=l x+m y+n t$,

$$
\begin{gathered}
u_{2}=-\frac{2 \sqrt{-\sigma} l^{\alpha}}{\sqrt{a} \operatorname{coth}_{\alpha}(-\sqrt{-\sigma} \xi)}+\frac{2 b l^{\alpha}-a m^{\alpha}}{a^{2} l^{\alpha}}, \\
v_{2}=-\frac{2 \sqrt{-\sigma} m^{\alpha}}{\sqrt{a} \operatorname{coth}_{\alpha}(-\sqrt{-\sigma} \xi)}+\frac{2 b m^{\alpha} l^{\alpha}-a m^{2 \alpha}}{a^{2} l^{2 \alpha}}+c,
\end{gathered}
$$

where $\sigma<0, \xi=l x+m y+n t$,

$$
\begin{gathered}
u_{3}=-\frac{2 \sqrt{\sigma} l^{\alpha}}{\sqrt{a} \tan _{\alpha}(\sqrt{\sigma} \xi)}+\frac{2 b l^{\alpha}-a m^{\alpha}}{a^{2} l^{\alpha}}, \\
v_{3}=-\frac{2 \sqrt{\sigma} m^{\alpha}}{\sqrt{a} \tan _{\alpha}(\sqrt{\sigma} \xi)}+\frac{2 b m^{\alpha} l^{\alpha}-a m^{2 \alpha}}{a^{2} l^{2 \alpha}}+c,
\end{gathered}
$$

where $\sigma>0, \xi=l x+m y+n t$,

$$
\begin{gathered}
u_{4}=\frac{2 \sqrt{\sigma} l^{\alpha}}{\sqrt{a} \cot _{\alpha}(\sqrt{\sigma} \xi)}+\frac{2 b l^{\alpha}-a m^{\alpha}}{a^{2} l^{\alpha}}, \\
v_{4}=\frac{2 \sqrt{\sigma} m^{\alpha}}{\sqrt{a} \cot _{\alpha}(\sqrt{\sigma} \xi)}+\frac{2 b m^{\alpha} l^{\alpha}-a m^{2 \alpha}}{a^{2} l^{2 \alpha}}+c,
\end{gathered}
$$

where $\sigma>0, \xi=l x+m y+n t$,

$$
u_{5}=\frac{2 \sigma l^{\alpha}\left(\omega+\xi^{\alpha}\right)}{\sqrt{a} \Gamma(1+a)}+\frac{2 b l^{\alpha}-a m^{\alpha}}{a^{2} l^{\alpha}},
$$

$$
v_{5}=\frac{2 \sigma m^{\alpha}\left(\omega+\xi^{\alpha}\right)}{\sqrt{a} \Gamma(1+a)}+\frac{2 b m^{\alpha} l^{\alpha}-a m^{2 \alpha}}{a^{2} l^{2 \alpha}}+c \text {, }
$$

where $\sigma=0, \xi=l x+m y+n t$. And $n=\left(\left(-12 a b m^{\alpha}+\right.\right.$ $\left.\left.9 a^{2} m^{2 \alpha} l^{-\alpha}+12 b^{2} l 6 \alpha-6 b a^{3} c l^{\alpha}+4 a^{3} l^{3 \alpha} \sigma\right) / 2 a^{2}\right)^{1 / \alpha}, l, m, c$, and $\omega$ are arbitrary constants.

From Cases 2, 3, 4, 5, 6, 7, and 8, we obtain many other exact solutions of (1). Here, we omit them for simplicity.

For $\alpha=1$, generalized hyperbolic function solutions and generalized trigonometric function solutions degrade into hyperbolic function solutions and trigonometric function solutions. We stress on the fact that when $\alpha \rightarrow 1$ these obtained exact solutions including solitary solutions and rational solutions give the ones of the standard form equation of the space-time fractional KD equation (1).

Example 2 (The space-time fractional NNV equations). By considering the traveling wave transformations $u=u(\xi)$, 
$v=v(\xi)$, and $\xi=l x+m y+n t$, (2) can be reduced to the following nonlinear fractional ODEs:

$$
\begin{gathered}
n^{\alpha} D_{\xi}^{\alpha} u=A l^{3 \alpha} D_{\xi}^{3 \alpha} u+B m^{3 \alpha} u D_{\xi}^{3 \alpha} u-3 A l^{\alpha} u D_{\xi}^{\alpha} v \\
-3 A l^{\alpha} v D_{\xi}^{\alpha} u-3 B m^{\alpha} u D_{\xi}^{\alpha} w-3 B m^{\alpha} w D_{\xi}^{\alpha} u \\
l^{\alpha} D_{\xi}^{\alpha} u=m^{\alpha} D_{\xi}^{\alpha} v \\
m^{\alpha} D_{\xi}^{\alpha} u=l^{\alpha} D_{\xi}^{\alpha} w .
\end{gathered}
$$

From (36)-(37) and using the definition of Jumarie's modified Riemann-Liouville derivative, one gets

$$
\begin{aligned}
& v=\frac{l^{\alpha}}{m^{\alpha}} u+c_{1}, \\
& w=\frac{m^{\alpha}}{l^{\alpha}} u+c_{2},
\end{aligned}
$$

where $c_{1}$ and $c_{2}$ are arbitrary constants. Substituting (38) into (35), one obtains

$$
\begin{aligned}
\left(n^{\alpha}+\right. & \left.6 A \frac{l^{2 \alpha}}{m^{\alpha}} u+3 A l^{\alpha} c_{1}+6 b \frac{m^{2 \alpha}}{l^{\alpha}} u+3 B m^{\alpha} c_{2}\right) \\
& \times D_{\xi}^{\alpha} u-\left(A l^{3 \alpha}+B m^{3 \alpha}\right) D_{\xi}^{3 \alpha} u=0 .
\end{aligned}
$$

By balancing the highest order derivative terms and nonlinear terms in (39), we suppose that (39) has the following formal solution:

$$
u(\xi)=b_{0}+b_{1} \varphi(\xi)+b_{2} \varphi^{2}(\xi)+\frac{b_{3}}{\varphi(\xi)}+\frac{b_{4}}{\varphi^{2}(\xi)} .
$$

Substituting (40) into (39) along with (14), and collecting the coefficients of $\varphi^{i}$ and setting them to be zero, we can get a set of algebraic equation about $l, m, n, c_{1}, c_{2}, b_{0}, b_{1}, b_{2}, b_{3}$, and $b_{4}$. Solving the algebraic equations by Mathematica, we have

Case 1. One has

$$
\begin{gathered}
b_{0}=\frac{m^{\alpha} l^{\alpha}\left(8 A l^{3 \alpha} \sigma+8 B m^{3 \alpha} \sigma-3 A c_{1} l^{\alpha}-3 B c_{2} m^{\alpha}-n^{\alpha}\right)}{6 A l^{3 \alpha}+6 B m^{3 \alpha}}, \\
b_{1}=0, \quad b_{2}=-2 m^{\alpha} l^{\alpha} \\
b_{3}=0, \quad b_{4}=2 m^{\alpha} l^{\alpha} \sigma^{2} .
\end{gathered}
$$

Case 2. One has

$$
\begin{gathered}
b_{0}=\frac{m^{\alpha} l^{\alpha}\left(8 A l^{3 \alpha} \sigma+8 B m^{3 \alpha} \sigma-3 A c_{1} l^{\alpha}-3 B c_{2} m^{\alpha}-n^{\alpha}\right)}{6 A l^{3 \alpha}+6 B m^{3 \alpha}}, \\
b_{1}=0, \quad b_{2}=0 \\
b_{3}=0, \quad b_{4}=2 m^{\alpha} l^{\alpha} \sigma^{2} .
\end{gathered}
$$

Case 3. One has

$$
\begin{gathered}
b_{0}=\frac{m^{\alpha} l^{\alpha}\left(8 A l^{3 \alpha} \sigma+8 B m^{3 \alpha} \sigma-3 A c_{1} l^{\alpha}-3 B c_{2} m^{\alpha}-n^{\alpha}\right)}{6 A l^{3 \alpha}+6 B m^{3 \alpha}} \\
b_{1}=0, \quad b_{2}=2 m^{\alpha} l^{\alpha} \\
b_{3}=0, \quad b_{4}=0 .
\end{gathered}
$$

Using Case 1, (40), and the solutions of (14), we can find the following exact solutions of NFDEs (2):

$$
\begin{aligned}
u_{1}= & -2 m^{\alpha} l^{\alpha} \sigma \tanh _{\alpha}^{2}(\sqrt{-\sigma} \xi)-\frac{2 m^{\alpha} l^{\alpha} \sigma}{\tanh _{\alpha}^{2}(\sqrt{-\sigma} \xi)} \\
& +\frac{m^{\alpha} l^{\alpha}\left(8 A l^{3 \alpha} \sigma+8 B m^{3 \alpha} \sigma-3 B c_{2} m^{\alpha}-3 A c_{1} l^{\alpha}-n^{\alpha}\right)}{6 A l^{3 \alpha}+6 B m^{3 \alpha}} \\
v_{1}= & -2 l^{2 \alpha} \sigma \tanh _{\alpha}^{2}(\sqrt{-\sigma} \xi)-\frac{2 l^{2 \alpha} \sigma}{\tanh _{\alpha}^{2}(\sqrt{-\sigma} \xi)} \\
& +\left(m ^ { \alpha } l ^ { \alpha } \left(8 A l^{5 \alpha} \sigma+8 B l^{2 \alpha} m^{3 \alpha} \sigma-3 B c_{2} l^{2 \alpha} m^{\alpha}\right.\right. \\
& \left.\quad-3 A c_{1} l^{3 \alpha}-l^{2 \alpha} n^{\alpha}\right) \\
w_{1}= & -2 m^{2 \alpha} \sigma \tanh _{\alpha}^{2}(\sqrt{-\sigma} \xi)-\frac{2 m^{2 \alpha} \sigma}{\tanh _{\alpha}^{2}(\sqrt{-\sigma} \xi)} \\
& +\left(m^{\alpha} l^{\alpha}\left(8 A l^{3 \alpha}+6 B l^{3 \alpha}\right)^{-1}+c_{1}^{2 \alpha} \sigma+8 B m^{5 \alpha} \sigma-3 B c_{2} m^{3 \alpha} m^{\alpha}\right. \\
& \left.\times\left(6 A l^{3 \alpha}+6 B m^{3 \alpha}\right)^{-1}\right)+c_{2}, \\
& \left.-3 A c_{1} l^{\alpha} m^{2 \alpha}-m^{2 \alpha} n^{\alpha}\right)
\end{aligned}
$$

where $\sigma<0, \xi=l x+m y+n t$,

$$
\begin{aligned}
u_{2}= & -2 m^{\alpha} l^{\alpha} \sigma \operatorname{coth}_{\alpha}^{2}(\sqrt{-\sigma} \xi)-\frac{2 m^{\alpha} l^{\alpha} \sigma}{\operatorname{coth}_{\alpha}^{2}(\sqrt{-\sigma} \xi)} \\
& +\frac{m^{\alpha} l^{\alpha}\left(8 A l^{3 \alpha} \sigma+8 B m^{3 \alpha} \sigma-3 B c_{2} m^{\alpha}-3 A c_{1} l^{\alpha}-n^{\alpha}\right)}{6 A l^{3 \alpha}+6 B m^{3 \alpha}}
\end{aligned}
$$

$$
\begin{aligned}
v_{2}=-2 l^{2 \alpha} \sigma \operatorname{coth}_{\alpha}^{2}(\sqrt{-\sigma} \xi)-\frac{2 l^{2 \alpha} \sigma}{\operatorname{coth}_{\alpha}^{2}(\sqrt{-\sigma} \xi)} \\
+\left(\left(8 A l^{5 \alpha} \sigma+8 B l^{2 \alpha} m^{3 \alpha} \sigma-3 B c_{2} l^{2 \alpha} m^{\alpha}\right.\right. \\
\left.-3 A c_{1} l^{3 \alpha}-l^{2 \alpha} n^{\alpha}\right) \\
\left.\times\left(6 A l^{3 \alpha}+6 B m^{3 \alpha}\right)^{-1}\right)+c_{1},
\end{aligned}
$$




$$
\begin{aligned}
w_{2}= & -2 m^{2 \alpha} \sigma \operatorname{coth}_{\alpha}^{2}(\sqrt{-\sigma} \xi)-\frac{2 m^{2 \alpha} \sigma}{\operatorname{coth}_{\alpha}^{2}(\sqrt{-\sigma} \xi)} \\
+ & \left(\left(8 A l^{3 \alpha} m^{2 \alpha} \sigma+8 B m^{5 \alpha} \sigma-3 B c_{2} m^{3 \alpha} m^{\alpha}\right.\right. \\
& \left.-3 A c_{1} l^{\alpha} m^{2 \alpha}-m^{2 \alpha} n^{\alpha}\right) \\
& \left.\times\left(6 A l^{3 \alpha}+6 B m^{3 \alpha}\right)^{-1}\right)+c_{2}
\end{aligned}
$$

where $\sigma<0, \xi=l x+m y+n t$,

$$
\begin{aligned}
& u_{3}= 2 m^{\alpha} l^{\alpha} \sigma \tan _{\alpha}^{2}(\sqrt{\sigma} \xi)+\frac{2 m^{\alpha} l^{\alpha} \sigma}{\tan _{\alpha}^{2}(\sqrt{\sigma} \xi)} \\
&+\frac{m^{\alpha} l^{\alpha}\left(8 A l^{3 \alpha} \sigma+8 B m^{3 \alpha} \sigma-3 B c_{2} m^{\alpha}-3 A c_{1} l^{\alpha}-n^{\alpha}\right)}{6 A l^{3 \alpha}+6 B m^{3 \alpha}} \\
& v_{3}=2 l^{2 \alpha} \sigma \tan _{\alpha}^{2}(\sqrt{\sigma} \xi)+\frac{2 l^{2 \alpha} \sigma}{\tan _{\alpha}^{2}(\sqrt{\sigma} \xi)} \\
&+\left(\left(8 A l^{5 \alpha} \sigma+8 B l^{2 \alpha} m^{3 \alpha} \sigma-3 B c_{2} l^{2 \alpha} m^{\alpha}\right.\right. \\
&\left.\quad-3 A c_{1} l^{3 \alpha}-l^{2 \alpha} n^{\alpha}\right) \\
&\left.\quad \times\left(6 A l^{3 \alpha}+6 B m^{3 \alpha}\right)^{-1}\right)+c_{1}, \\
& w_{3}=2 m^{2 \alpha} \sigma \tan _{\alpha}^{2}(\sqrt{\sigma} \xi)+\frac{2 m^{2 \alpha} \sigma}{\tan _{\alpha}^{2}(\sqrt{\sigma} \xi)} \\
&+(\left(8 A l^{3 \alpha} m^{2 \alpha} \sigma+8 B m^{5 \alpha} \sigma-3 B c_{2} m^{3 \alpha} m^{\alpha}\right. \\
&\left.\quad-3 A c_{1} l^{\alpha} m^{2 \alpha}-m^{2 \alpha} n^{\alpha}\right) \\
&\left.\times\left(6 A l^{3 \alpha}+6 B m^{3 \alpha}\right)^{-1}\right)+c_{2},
\end{aligned}
$$

where $\sigma>0, \xi=l x+m y+n t$

$$
\begin{aligned}
& u_{4}= 2 m^{\alpha} l^{\alpha} \sigma \cot _{\alpha}^{2}(\sqrt{\sigma} \xi)+\frac{2 m^{\alpha} l^{\alpha} \sigma}{\cot _{\alpha}^{2}(\sqrt{\sigma} \xi)} \\
&+\frac{m^{\alpha} l^{\alpha}\left(8 A l^{3 \alpha} \sigma+8 B m^{3 \alpha} \sigma-3 B c_{2} m^{\alpha}-3 A c_{1} l^{\alpha}-n^{\alpha}\right)}{6 A l^{3 \alpha}+6 B m^{3 \alpha}} \\
& v_{4}=2 l^{2 \alpha} \sigma \cot _{\alpha}^{2}(\sqrt{\sigma} \xi)+\frac{2 l^{2 \alpha} \sigma}{\cot _{\alpha}^{2}(\sqrt{\sigma} \xi)} \\
&+\left(\left(8 A l^{5 \alpha} \sigma+8 B l^{2 \alpha} m^{3 \alpha} \sigma-3 B c_{2} l^{2 \alpha} m^{\alpha}\right.\right. \\
&\left.\quad-3 A c_{1} l^{3 \alpha}-l^{2 \alpha} n^{\alpha}\right) \\
&\left.\quad \times\left(6 A l^{3 \alpha}+6 B m^{3 \alpha}\right)^{-1}\right)+c_{1}
\end{aligned}
$$

$$
\begin{aligned}
w_{4}= & 2 m^{2 \alpha} \sigma \cot _{\alpha}^{2}(\sqrt{\sigma} \xi)+\frac{2 m^{2 \alpha} \sigma}{\cot _{\alpha}^{2}(\sqrt{\sigma} \xi)} \\
+ & \left(\left(8 A l^{3 \alpha} m^{2 \alpha} \sigma+8 B m^{5 \alpha} \sigma-3 B c_{2} m^{3 \alpha} m^{\alpha}\right.\right. \\
& \left.-3 A c_{1} l^{\alpha} m^{2 \alpha}-m^{2 \alpha} n^{\alpha}\right) \\
& \left.\times\left(6 A l^{3 \alpha}+6 B m^{3 \alpha}\right)^{-1}\right)+c_{2}
\end{aligned}
$$

where $\sigma>0, \xi=l x+m y+n t$,

$$
\begin{aligned}
u_{5}= & \frac{2 \sigma^{2} l^{\alpha} m^{\alpha}\left(\omega+\xi^{\alpha}\right)^{2}}{\Gamma^{2}(1+a)}+\frac{2 l^{\alpha} m^{\alpha} \Gamma^{2}(1+a)}{\left(\omega+\xi^{\alpha}\right)^{2}} \\
& +\frac{m^{\alpha} l^{\alpha}\left(8 A l^{3 \alpha} \sigma+8 B m^{3 \alpha} \sigma-3 B c_{2} m^{\alpha}-3 A c_{1} l^{\alpha}-n^{\alpha}\right)}{6 A l^{3 \alpha}+6 B m^{3 \alpha}}, \\
v_{5}= & \frac{2 \sigma^{2} l^{\alpha}\left(\omega+\xi^{\alpha}\right)^{2}}{\Gamma^{2}(1+a)}+\frac{2 l^{2 \alpha} \Gamma^{2}(1+a)}{\left(\omega+\xi^{\alpha}\right)^{2}} \\
& +\left(\left(8 A l^{5 \alpha} \sigma+8 B l^{2 \alpha} m^{3 \alpha} \sigma-3 B c_{2} m^{\alpha} l^{2 \alpha}-3 A c_{1} l^{3 \alpha}\right.\right. \\
& \left.\quad-l^{2 \alpha} n^{\alpha}\right) \\
& \left.\times\left(6 A l^{3 \alpha}+6 B m^{3 \alpha}\right)^{-1}\right)+c_{1}, \\
w_{5}= & \frac{2 \sigma^{2} l^{\alpha}\left(\omega+\xi^{\alpha}\right)^{2}}{\Gamma^{2}(1+a)}+\frac{2 l^{2 \alpha} \Gamma^{2}(1+a)}{\left(\omega+\xi^{\alpha}\right)^{2}} \\
& +\left(\left(8 A l^{3 \alpha} m^{2 \alpha} \sigma+8 B l^{5 \alpha} m^{3 \alpha} \sigma-3 B c_{2} m^{3 \alpha}\right.\right. \\
& \left.\quad-3 A c_{1} l^{\alpha} m^{2 \alpha}-m^{2 \alpha} n^{\alpha}\right) \\
& \left.\times\left(6 A l^{3 \alpha}+6 B m^{3 \alpha}\right)^{-1}\right)+c_{2},
\end{aligned}
$$

where $\sigma=0, \xi=l x+m y+n t$. And $l, m, c$, and $\omega$ are arbitrary constants, $\tanh _{\alpha}, \operatorname{coth}_{\alpha}, \tan _{\alpha}$, and $\cot _{\alpha}$ are generalized hyperbolic and trigonometric functions.

From Cases 2 and 3, we obtain many other exact solutions of (2). Here, we omit them for simplicity, too.

As $\alpha \rightarrow 1$, solutions (44)-(48) obtained above become the ones of the standard form equation of the NNV model, and the solutions cannot be directly constructed by other methods.

\section{Conclusion}

In the paper, based on the relationship of the fractional coupled equations and the properties of the Jumarie's modified Riemann-Liouville derivative, we proposed a new generalized fractional subequation method to construct exact solutions of space-time fractional coupled differential equations. In order to illustrate the validity and advantages of the algorithm, we 
apply it to space-time fractional coupled KonopelchenkoDubrovsky equations and Nizhnik-Novikov-Veselov equations. As a results, many exact solutions are obtained. The results show that this new generalized fractional subequations method is direct, effective, and can be used for many other fractional coupled differential equations.

\section{Acknowledgment}

The authors express their thanks to the referees for their fruitful advice and comments.

\section{References}

[1] I. Podlubny, Fractional Differential Equations, vol. 198 of Mathematics in Science and Engineering, Academic Press, San Diego, Calif, USA, 1999.

[2] R. Metzler and J. Klafter, "The random walk's guide to anomalous diffusion: a fractional dynamics approach," Physics Reports, vol. 339, no. 1, p. 77, 2000.

[3] K. M. Kolwankar and A. D. Gangal, "Local fractional FokkerPlanck equation," Physical Review Letters, vol. 80, no. 2, pp. 214217, 1998.

[4] G. C. Wu, "A fractional variational iteration method for solving fractional nonlinear differential equations," Computers \& Mathematics with Applications, vol. 61, no. 8, pp. 2186-2190, 2011.

[5] H. G. Sun and W. Chen, "Fractal derivative multi-scale model of fluid particle transverse accelerations in fully developed turbulence," Science in China, Series E, vol. 52, no. 3, pp. 680683, 2009.

[6] W. Chen and H. G. Sun, "Multiscale statistical model of fullydeveloped turbulence particle accelerations," Modern Physics Letters B, vol. 23, no. 3, pp. 449-452, 2009.

[7] J. Cresson, "Scale calculus and the Schrödinger equation," Journal of Mathematical Physics, vol. 44, no. 11, pp. 4907-4938, 2003.

[8] G. Jumarie, "Modified Riemann-Liouville derivative and fractional Taylor series of nondifferentiable functions further results," Computers \& Mathematics with Applications, vol. 51, no. 9-10, pp. 1367-1376, 2006.

[9] S. W. Wang and M. Y. Xu, "Axial Couette flow of two kinds of fractional viscoelastic fluids in an annulus," Nonlinear Analysis. Real World Applications, vol. 10, no. 2, pp. 1087-1096, 2009.

[10] X. Y. Jiang and M. Y. Xu, "The time fractional heat conduction equation in the general orthogonal curvilinear coordinate and the cylindrical coordinate systems," Physica A, vol. 389, no. 17, pp. 3368-3374, 2010.

[11] Y.-Q. Liu and J.-H. Ma, "Exact solutions of a generalized multifractional nonlinear diffusion equation in radical symmetry," Communications in Theoretical Physics, vol. 52, no. 5, pp. 857861, 2009.

[12] J. H. Ma and Y. Q. Liu, "Exact solutions for a generalized nonlinear fractional Fokker-Planck equation," Nonlinear Analysis. Real World Applications, vol. 11, no. 1, pp. 515-521, 2010.

[13] Y. Q. Liu, "Approximate solutions of fractional nonlinear equations using homotopy perturbation transformation method," Abstract and Applied Analysis, vol. 2012, Article ID 752869, 14 pages, 2012.

[14] Y. Q. Liu, "Study on space-time fractional nonlinear biological equation in radial symmetry," Mathematical Problems in Engineering, vol. 2013, Article ID 654759, 6 pages, 2013.
[15] Y. Q. Liu, "Variational homotopy perturbation method for solving fractional initial boundary value problems," Abstract and Applied Analysis, vol. 2012, Article ID 727031, 10 pages, 2012.

[16] H. A. Ghany and M. S. Mohammed, "White noise functional solutions for Wick-type stochastic fractional KdV-BurgersKuramoto equations," Chinese Journal of Physics, vol. 50, no. 4, pp. 619-627, 2012.

[17] M. L. Wang, "Solitary wave solutions for variant Boussinesq equations," Physics Letters A, vol. 199, no. 3-4, pp. 169-172, 1995.

[18] S. Zhang and H.-Q. Zhang, "Fractional sub-equation method and its applications to nonlinear fractional PDEs," Physics Letters A, vol. 375, no. 7, pp. 1069-1073, 2011.

[19] H. Jafari, H. Tajadodi, N. Kadkhoda, and D. Baleanu, "Fractional sub-equation method for Cahn-Hilliard and KleinGordon equations," Abstract and Applied Analysis, vol. 2012, Article ID 587179, 5 pages, 2013.

[20] B. Tang, Y. He, L. Wei, and X. Zhang, "A generalized fractional sub-equation method for fractional differential equations with variable coefficients," Physics Letters A, vol. 376, no. 38-39, pp. 2588-2590, 2012.

[21] S. M. Guo, L. Q. Mei, Y. Li, and Y. Sun, “The improved fractional sub-equation method and its applications to the space-time fractional differential equations in fluid mechanics," Physics Letters A, vol. 376, no. 4, pp. 407-411, 2012.

[22] J. P. Zhao, B. Tang, S. Kumar, and Y. R. Hou, "The extended fractional subequation method for nonlinear fractional differential equations," Mathematical Problems in Engineering, vol. 2012, Article ID 924956, 11 pages, 2012.

[23] H. A. Ghany, "Exact solutions for stochastic generalized hirotasatsuma coupled KdV equations," Chinese Journal of Physics, vol. 49, no. 4, pp. 926-940, 2011.

[24] A. H. Ghany, A. S. O. El Bab, A. M. Zabel, and A.-A. Hyder, "The fractional coupled Kdv equations: exact solutions and white noise functional approach," Chinese Physics B, vol. 22, no. 8, Article ID 080501.

[25] S. Zhang, "Exp-function method for Riccati equation and new exact solutions with two arbitrary functions of $(2+1)$ dimensional Konopelchenko-Dubrovsky equations," Applied Mathematics and Computation, vol. 216, no. 5, pp. 1546-1552, 2010.

[26] B.-C. Shin, M. T. Darvishi, and A. Barati, "Some exact and new solutions of the Nizhnik-Novikov-Vesselov equation using the Exp-function method," Computers \& Mathematics with Applications, vol. 58, no. 11-12, pp. 2147-2151, 2009. 


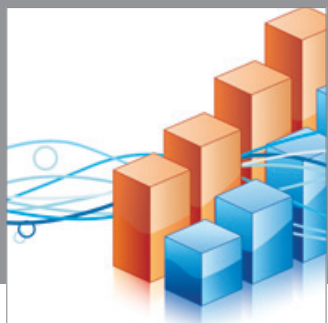

Advances in

Operations Research

mansans

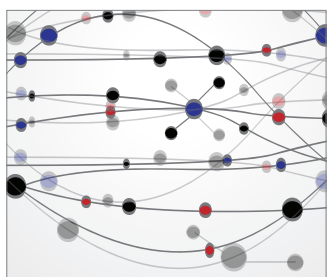

The Scientific World Journal
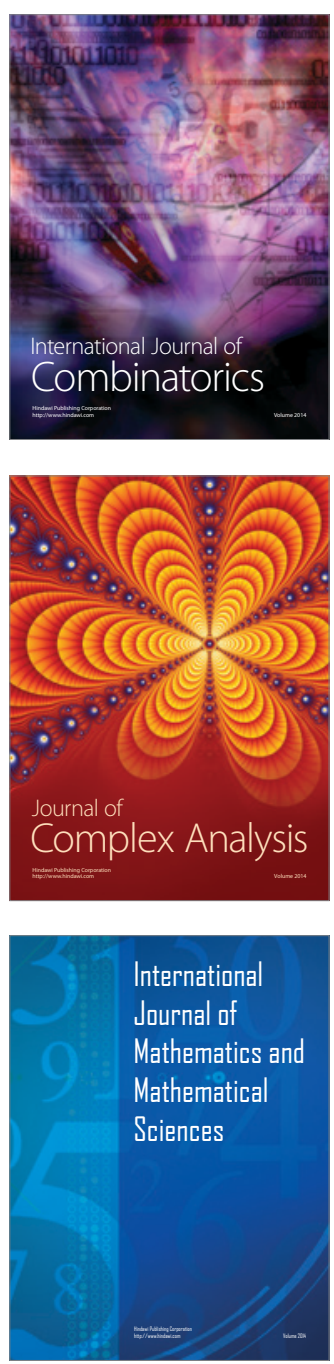
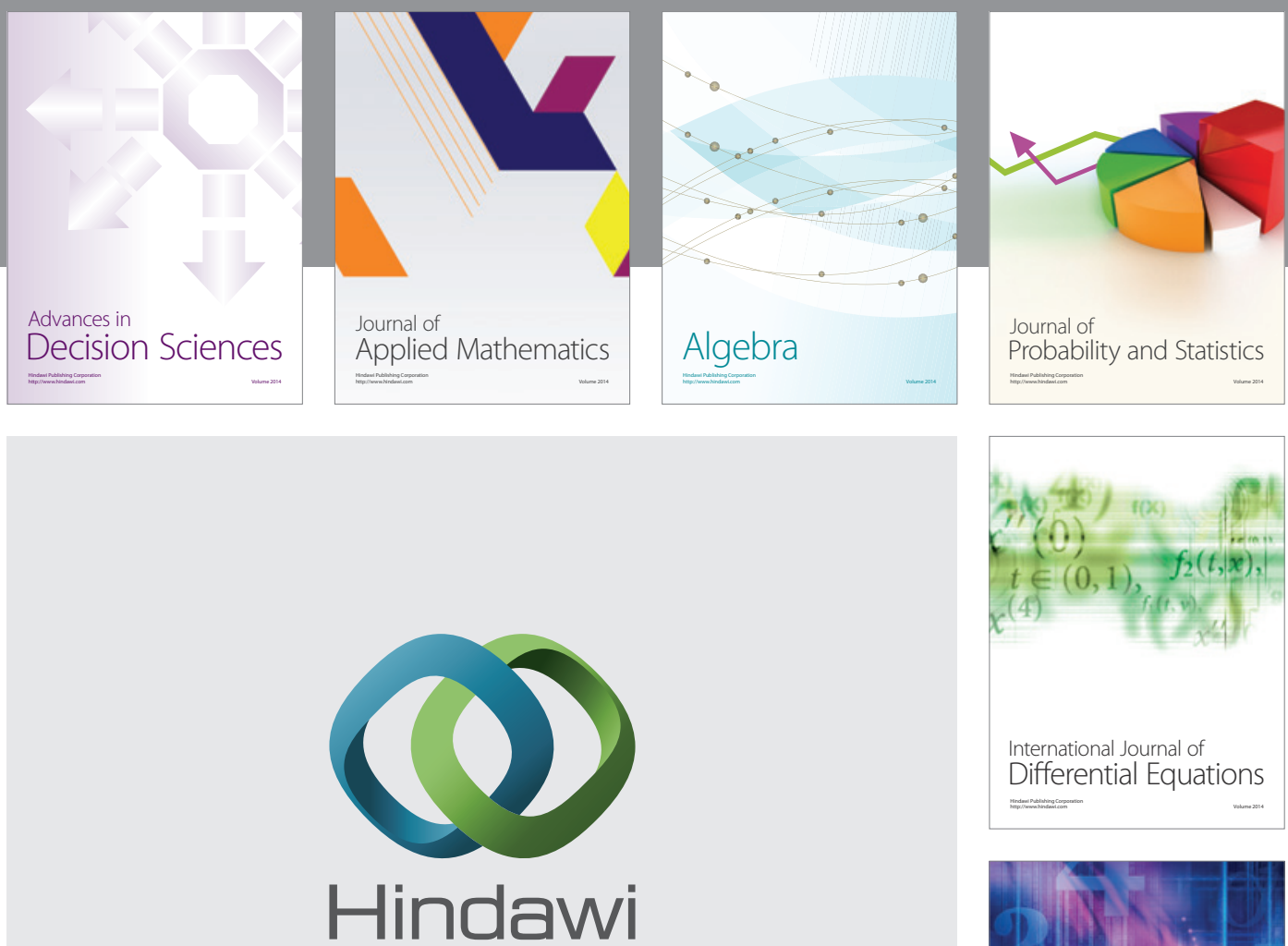

Submit your manuscripts at http://www.hindawi.com
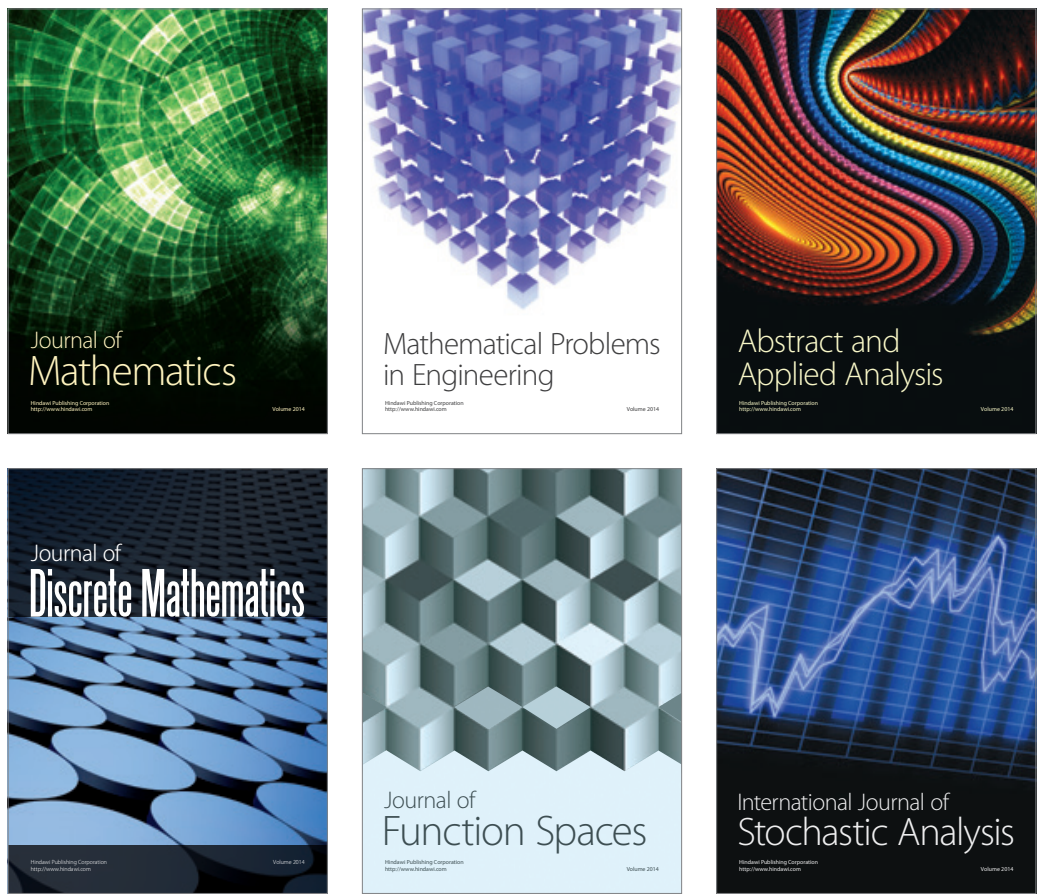

Journal of

Function Spaces

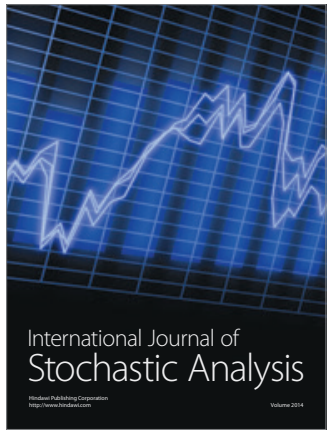

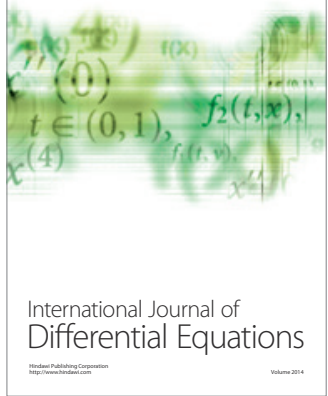
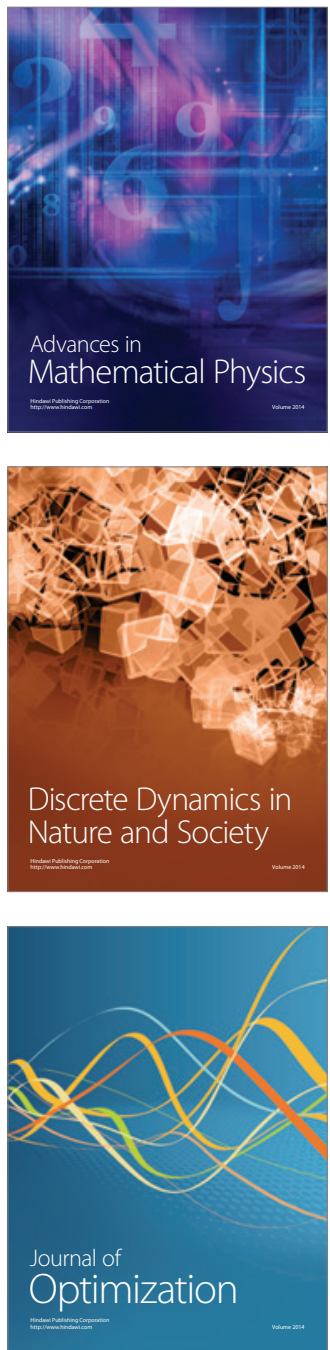